\title{
Global Kinematics \\ for Manipulator Planning and Control
}

\author{
Joel W. Burdick \\ Dept. of Mechanical Engineering, California Institute of Technology
}

\begin{abstract}
This paper reviews one method of global kinematic analysis, based on a manifold mapping reformulation of manipulator kinematics, which is suitable for both non-redundant and redundant serial chain manipulators. Two applications of this approach are considered: the relationship between changing pose and singularities for non-redundant manipulators and the homotopy class of redundant manipulator self-motions. The first relationship has important implications for nonredundant manipulator regrasping operations. Variations in redundant manipulator self-motion homotopy can have an important effect on motion and redundancy resolution planning, since they can lead to algorithmic singularities and unexpected loss of manipulator capabilities.
\end{abstract}

\section{Introduction}

Robotic control and motion planning contains a strong geometric and kinematic component. Motion planning in complicated environments or with complicated goals is a global problem, and consequently, kinematic analysis techniques which are global in their scope are required for intelligent planning of manipulator motions and efficient use of their capabilities. To understand complicated kinematic phenomena, manipulator kinematic researchers have used powerful geometric and topological methods to analyze manipulator kinematic properties and kinematic algorithms $[3,8,13]$. These global methods are particularly well suited to the analysis of comparatively complicated redundant manipulator kinematics. Consequently, much work in global kinematics has focused on redundant manipulators and the additional kinematic subtleties which arise from the extra degrees of freedom $[6,13,15]$.

This paper reviews one such global approach to manipulator kinematic analysis which is based on a manifold mapping reformulation of manipulator kinematics. The joint space and workspace are interpreted as manifolds which have further structure imposed by the geometry and properties of kinematic singularities. This approach is used in this paper to investigate two global kinematic phenomenon. First, we consider the ability of non-redundant regional manipulators with three revolute joints (3R manipulators) to change pose (move from one inverse kinematic solution to another) without encountering a singularity. Second, we consider the homotopy classes of redundant manipulator self-motions and their variations throughout the workspace. In some cases, these variations can lead to algorithmic singularities and un- expected loss or reduction of redundancy resolution capabilities. Much of the work in this paper is based on $[5,7]$.

\section{A Manifold Mapping Reformulation of Manipula- tor Kinematics}

Only revolute jointed manipulators will be considered in this paper, although other manipulators can be similarly treated. A manipulator forward kinematic function, $f$, is a nonlinear vector function which relates a set of $n$ joint coordinates, $\bar{\theta}$, to a set of $m$ end-effector coordinates:

$$
\mathbf{x}=f(\bar{\theta}) .
$$

To globally analyze manipulator kinematics, it is useful to rephrase the forward and inverse kinematic problems in terms of manifold mappings. From a point-wise mapping perspective, $f$ maps a joint configuration, $\bar{\theta}$, to an end-effector location: $\mathbf{x}=f(\tilde{\theta})$. The set of all possible joint configurations forms a space, termed the "joint space" or "configuration space," which has a simple manifold structure. Similarly, the set of all possible end-effector locations forms the workspace, which is also a manifold.

The manifold structure of the joint space arises as follows. Let $\theta_{j}$ denote the joint rotation angle for the $j^{\text {th }}$ revolute joint. If the motion of the $j^{\text {th }}$ joint is not limited due to mechanical reasons, $\theta_{j}$ can take on all values in the interval $[-\pi, \pi]$. However, $\pi$ and $-\pi$, are equivalent, and their identification yields a circle, denoted by $S^{1}$ in Figure 1 . The configuration space, $\mathcal{C}$, of an $n$-revolute-jointed manipulator is the $n$-times product of the individual joint manifolds:

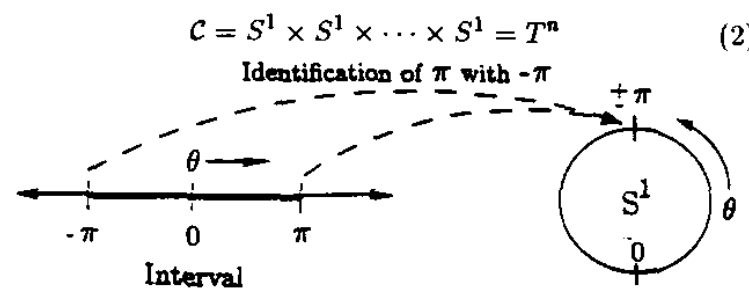

Figure 1: Configuration Space of a Revolute Joint where $T^{n}$ is an $n$-torus. Each of the circles that make up the torus is termed a generator of the torus, and is physically equivalent to a $2 \pi$ rotation of one joint. There is a one-toone correspondence between each point in $\mathcal{C}$ and a distinct manipulator configuration. For example, the $2 R$ planar manipulator in Figure 2(a) has a 2-torus configuration space 
(Figure 2(b)). While $\mathcal{C}$ is properly a torus, there are other useful representations of $\mathcal{C}$. For example, $\mathcal{C}$ in Figure 2 can be presented as a square with dimension $2 \pi$ by "cutting" the torus along two generators (Figure 3 ). The 3 -torus configuration space of a $3 R$ manipulator can be presented as a cube of dimensions $2 \pi$ by cutting along the 3 -tori generators, and the cubes in the following figures are 3-tori sliced along their generators.
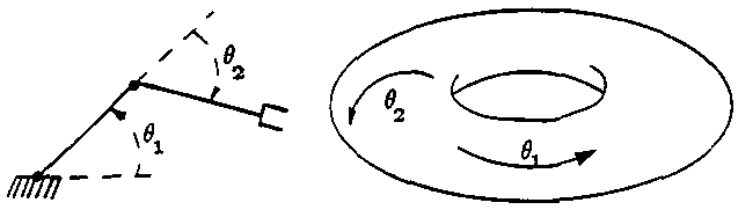

(a) Planar 2R Manipulator

(b) 2-torus Configuration Space Manifoid

Figure 2: Configuration Space of a 2-R Manipulator

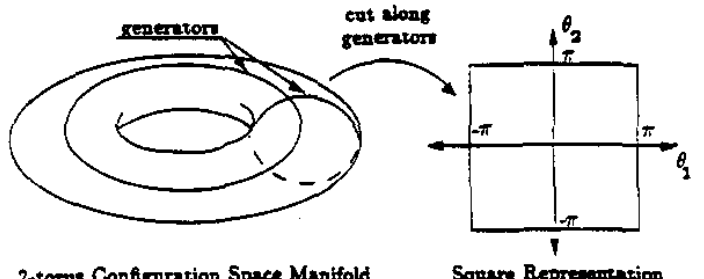

2-torws Confrorntion Space Manifold

Squere Repreverretion

Figure 3: Square Representation of a 2-Torus

Attach a frame to the manipulator end-effector. The manipulator's workspace manifold, $\mathcal{W}$, is the set of all possible locations and orientations of this frame as the manipulator joints are swept through all points of $\mathcal{C}$.

Rather than viewing $f$ as a point-wise mapping, one can alternatively view the action of $f$ as the global rearrangement of the configuration space manifold to produce the workspace manifold:

$$
f(\bar{\theta}): \mathcal{C} \rightarrow \mathcal{W} .
$$

Roughly speaking, the forward kinematic map "folds" the configuration space manifold into sheets along the loci of kinematic singularities; distorts each sheet; and combines the distorted sheets to form $\mathcal{W}$. The nature of this folding strongly effects global kinematic properties, and in turn, the global folding action of the forward kinematic map is strongly governed by the properties of kinematic singularities [6]. In the following section, this folding concept and the effect of singularities upon kinematics mappings will be used to analyze the relationship between pose and singular configurations.

\section{3R Manipulator Poses and Singularities}

For non-redundant manipulators, there is more than one solution to the inverse kinematic problem: $\bar{\theta}=f^{-1}(\mathbf{x})$. Each of these solutions is termed a "pose," or a different physical configuration of the manipulator which places the endeffector in the same position and orientation. In this section we consider the question: can a manipulator (in this case a $3 \mathrm{R}$ manipulator) change pose without passing through a singularity? This information is important for regrasping and cyclic motion operations. In performing a highly dextrous task (which may be sensory based, and therefore not planned offline), a manipulator might near its joint limits while following a trajectory. To continue its motion without encountering joint limits, the manipulator could change pose to place its joints in another configuration farther away from the joint limits and resume its trajectory. Changing pose might also be necessary for tasks, such as turning a screw, where regrasping of the object via a pose change is necessary.

It has previously been assumed [4] that non-redundant revolute-jointed manipulators must pass through a singular configuration when changing pose. However, a counter example to this notion will be given below. Some manipulators must indeed pass through a singularity while changing pose, yet it can shown than many manipulators need not encounter a singularity while changing pose. This is an important global kinematic property that can be naturally interpreted in the kinematic framework reviewed in Section 2.

The geometric and topological properties of a $3 \mathrm{R}$ manipulator's singularities determine its ability to change pose in a non-singular manner. It can be shown [5] that the loci of $3 R$ manipulator critical ${ }^{1}$ points form continuous two-

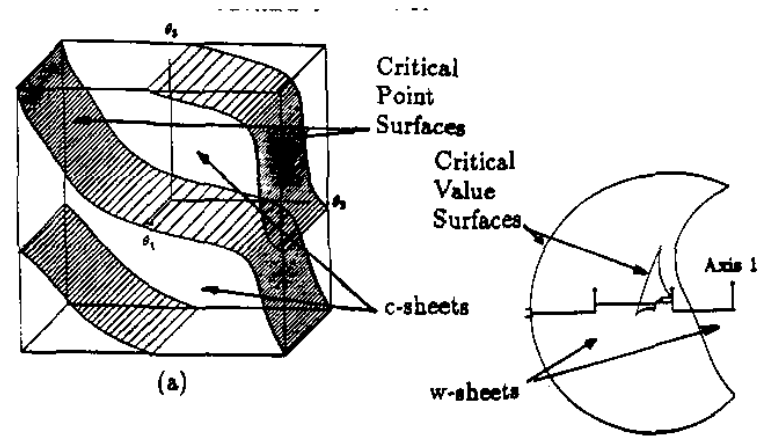

(b)

Figure 4: 3R Manipulator Configuration Space and Workspace

dimensional surfaces in $\mathcal{C}$. These surfaces divide $\mathcal{C}$ into disjoint regions, which are termed configuration space sheets, hereafter shortened to c-sheets. For example, Figure 4(a) shows the configuration space torus and critical point surfaces for a $3 \mathrm{R}$ manipulator with kinematic parameters: $\alpha_{1}=$ $60^{\circ}, \alpha_{2}=-90^{\circ}, a_{1}=1.3, a_{2}=1.7, a_{3}=1.6, d_{1}=0.4$, $d_{3}=-0.2$. In this case, the singularities divide $\mathcal{C}$ into two disjoint c-sheets.

\footnotetext{
1 For a non-redundant manipulatos, a regular point of $f$ is a conflguv. sation, $\bar{\theta}$, for which $f(\bar{\theta})$ is not singular (the Jacobian of $f$ is full rank). A regular value is an end-effector location $x=f(\bar{\theta})$ where $\bar{\theta}$ is a regular point. A critical point is a conflguration, $\bar{B}$, such that $f(\bar{\theta})$ is aingular. A critical value is an end-effector locstion $x=f(\bar{\theta})$ where $\bar{\theta}$ is a critical point. For redundant manipulatore, these definitions are extended $s 0$ that a regular value has all regular points in ita preimage, a critical value has all criticsl points in ita preimage, and a coregular value is an $x$ which has both regular and critical points in its preimage.
} 
Each c-sheet, which is a submanifold of $\mathcal{C}$, is mapped to a subregion of $W$. Let the mapping of a $c$-sheet under the action of the restricted forward kinematic map be termed a workspace sheet, or w-sheet. The action of $f$ can be conceptually considered as a multi-step process. First, $f$ folds $\mathcal{C}$ along the critical point surfaces. The regions between the folds, the c-sheets, are distorted to form $w$-sheets. The wsheets are then identified to form $\mathcal{W}$. Figure 4(b) shows a cross-section (actually half of the cross-section symmetric about joint axis 1) of the workspace for the manipulator in Figure 4(a).

The ability to change pose with or without encountering a singularity can be phrased as a strictly topological question: is the mapping of the c-sheet to a w-sheet a one-to-one or many-to-one mapping. It can be shown [6] that a c-sheet and the restriction of $f$ to the $c$-sheet form a covering space [11] for a w-sheet. The question at hand can be phrased as follows: is the restriction of $f$ to a c-sheet a single sheeted or multiply-sheeted covering space for a w-sheet?

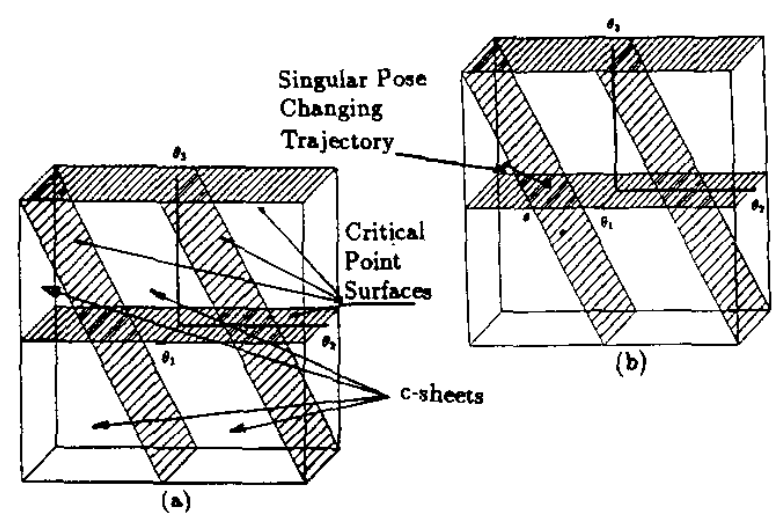

Figure 5: Elbow Manipulator Configuration Space and Critical Point Surfaces

If the c-sheet to w-sheet mapping is one-to-one, for a given regular end-effector location there can exist at most one inverse kinematic solution per $c$-sheet. In changing from one pose to another, the manipulator must follow a trajectory in $\mathcal{C}$ which passes from one $\mathrm{c}$-sheet to another. Since the c-sheets are bounded by critical point surfaces, this trajectory necessarily crosses a critical surface in $\mathcal{C}$. For example, Figure $5(a)$ shows the configuration space and critical points surfaces of an elbow manipulator with kinematic parameters: $a_{1}=0, a_{2}=1.0, a_{3}=1.0, \alpha_{1}=\pi / 2, \alpha_{2}=0$, $d_{2}=d_{3}=0$. There are always four inverse kinematic solutions for any given end-effector location, one per c-sheet. The inverse kinematic Solutions which place the end-effector at $(x, y, z)=(1.0,1.0,1.0)$ are shown in Figure 5(b). Any trajectory in $\mathcal{C}$ which connects two of these poses must intersect a critical point surface.

If the c-sheet to $w$-sheet mapping is many-to-one, more than one inverse kinematic solution can lie in a c-sheet. A tra. jectory which connects two inverse kinematic solutions in a c-sheet will not cross a singular boundary. Consequently, such a manipulator can change pose without passing through a singularity. Of course, one could pick a trajectory which crosses a critical point surface if desired.

For example, Figure 6 shows the configuration space of a $3 \mathrm{R}$ manipulator with kinematic parameters $\alpha_{1}=10^{\circ}, \alpha_{2}=$ $75^{\circ}, a_{1}=3.5, a_{2}=2.0, a_{3}=1.75, d_{2}=1.0, d_{3}=0.5$. The four inverse kinematic solutions which place the endeffector at $(x, y, z)=(3.5,0.0,0.85)$ are superimposed. It is easy to see that there are two inverse kinematic solutions in each c-sheet, and it is possible to connect these poses with a trajectory which does not intersect a critical point surface. It has been numerically verified that the trajectories in Figure 6 are indeed non-singular pose changing motions.

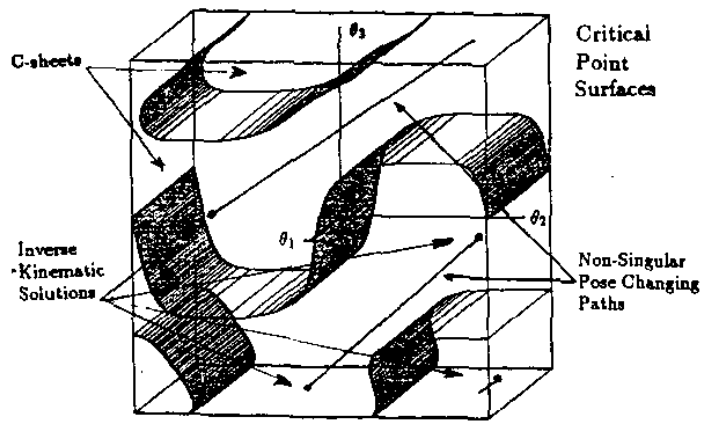

Figure 6: Non-Singular Pose Changing Trajectories of a 3R Manipulator

The above two examples show two different mapping behaviors for $3 \mathrm{R}$ manipulators with different geometries. What determines a manipulator's ability to change pose with or without encountering a singularity? It can be shown that for $3 \mathrm{R}$ manipulators, the ability to change pose without passing through a singularity is a function of the manipulator's kinematic geometry [5]. In [5] the concept of generic kinematic functions, introduced by Pai [12], is used to categorize all possible 3R manipulator geometries into generic and non-generic classes. In brief, generic $3 \mathrm{R}$ manipulators have critical point surfaces which form proper submanifolds of $\mathcal{C}$, and are necessarily disjoint. Conversely, it can be shown [5] that the critical point surfaces of non-generic manipulators intersect (as in Figure 5), typically dividing $\mathcal{C}$ into 4 or more $c$-sheets. For most non-generic manipulators, the basic geometry and topology of the forward mapping of non-generic $c$-sheets requires a one-to-one c-sheet to $\mathrm{w}$-sheet mapping relation on non-generic c-sheets [5].

Remark: Manipulators which always pass through a singular configuration when changing pose are non-generic manipulators.

Non-genericity is a necessary, but not sufficient condition to enforce a manipulator to encounter a singularity while changing pose. Some non-generic manipulators can change pose without passing through a singularity. That is, there are some non-generic manipulators which can change pose without encountering a singularity. In the space of all possible 3R manipulator geometries, non-generic manipulators form a set of measure zero. However, all industrial manipu- 
lators are non-generic, since it is shown in [5] that sucb common geometric features as parallel and intersecting axes lead to non-gencricity in $3 R$ manipulators. Consequently, industrial 3R regional manipulators encounter singularities when changing pose. Since most industrial manipulators have a $3 R$ regional structure with a $3 R$ wrist attached, these results hold for all $6 \mathrm{R}$ manipulators with wrists as well

Pose changing is an important capability for manipulators which must perform highly dextrous tasks in the presence of obstacles and joint limits. Consequently, motion planners and control algorithms must be able to plan and control trajectories which encounter singularities in order to take advantage of the opportunities afforded by pose changing. Conversely, one may wish to design and use non-generic manipulators for tasks where pose changing is often required.

\section{Redundant Manipulator Self-Motions}

While non-redundant manipulators have a finjte number of discrete solutions to the inverse kinematic problem, redundant manipulators have an infinity of solutions. The set of solutions and their properties can be conveniently interpreted within the framework of Section 2. Let $r=(n-m)$ be the relative degrees of redundancy. Since $f$ is a smooth function which maps $\mathcal{C}$, a compact manifold, to $\mathcal{W}$, a redundant manipulator inverse kinematic solution for a regular value of $\mathbf{x}$ must be an $r$-dimensional submanifold of $\mathcal{C}[9]$. However, the preimage submanifold may be divided into more than one disjoint manifold. That is, a redundant inverse kinematic solution is the union of disjoint $r$-dimensional manifolds:

$$
f^{-1}(\mathbf{x})=\bigcup_{i}^{n_{s m}} M_{i}
$$

where $M_{i}$ is the $i^{\text {th }} r$-dimensional manifold in the inverse kinematic preimage, and $M_{i} \cap M_{j}=\emptyset$ when $i \neq j$. Each of the preimage manifolds physically corresponds to a "selfmotion," which is a continuous motion of the manipulator joints which leaves the cnd-effector motionless. Each $M_{i}$ is termed a self-motion manifold. $n_{s m}$ is the number of selfmotions in the preimage. Some properties of redundant manipulator self-motions, including bounds on the number of self-motions and their geometry, have been considered in [7].

The self-motion manifolds are best illustrated using two examples: a planar 3R manipulator (Figure 7) which is redundant with respect to the position of its end-effector, and a $4 \mathrm{R}$ regional manipulator which is similar to an "elbow" manipulator (Figure 8). The details of computing the $3 \mathrm{R}$ and $4 \mathrm{R}$ manipulator self-motion manifolds can be found in [7]. Figure 7 shows the self-motion manifolds of $3 R$ planar manipulator (embedded in the configuration space 3-torus) for two different locations of the end-effector. The inverse image of Point 1 contains two distinct self-motion manifolds (which appear as non-closed curves because of the cubic 3-torus representation), while the inverse image of Point 2 contains only one self-motion. The two distinct self-motion manifolds in the preimage of Point 1 physically corresponds to "up elbow" and "down elbow" self-motions which are an analogous generalization of the "up elbow" and "down elbow" configurations of a non-redundant two-link manipulator. In both cases, the self-motion manifolds are diffeomorphic to a circle. However, the preimage of Point 1 contains a $2 \pi$ joint rotation while the other preimage does not.

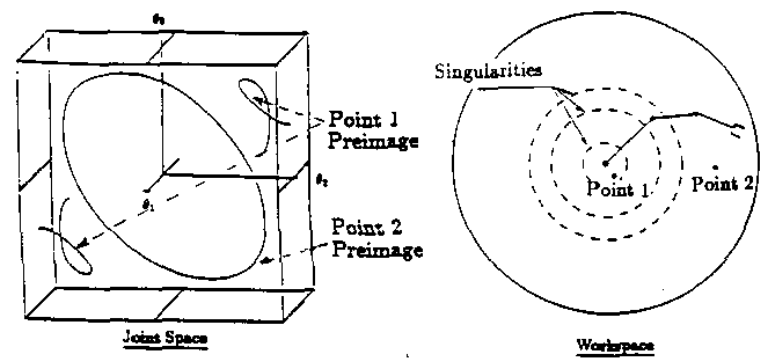

Figure 7: 3R Manipulator Self-Motions

The kinematic parameters of the $4 R$ manipulator in Figure 8 are: $\alpha_{0}=0 ; \alpha_{1}=\alpha_{2}=\pi / 2 ; \alpha_{3}=-\pi / 2 ; a_{0}=a_{1}=a_{2}=0$; $a_{3}=a_{4}=1.0 ; d_{1}=d_{2}=d_{3}=d_{4}=0$. For regular values of the end-effector, this manipulator has four distinct selfmotions. Each of the self-motions corresponds to a rotation of the plane containing links 3 and 4 about a line connecting the end-effector and the intersection of joint axes 1,2 , and 3 . Figure 9 shows the cubic representation of the projection of these four self-motions onto the $\theta_{1}-\theta_{2}-\theta_{3}$ and $\theta_{2}-\theta_{3}-\theta_{4} 3$-tori when the end-effector is located at $(x, y, z)=(0.0,1.0,0.9)$.

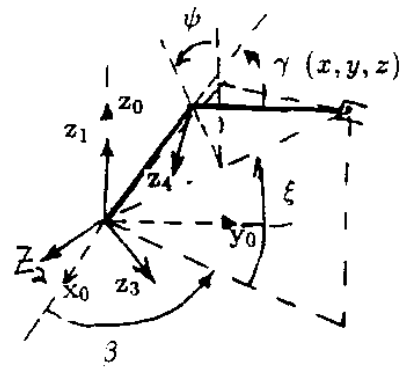

Figure 8: 4R Elbow-Like Manipulator
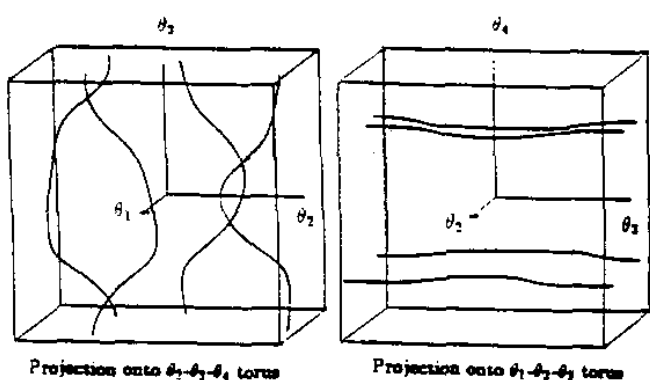

Figure 9: 4R Manipulator Self-Motion Manifolds

\subsection{Self-motion Homotopy Classes}

In Figure 7, the self-motion manifolds over Point 1 included a $2 \pi$ joint rotation, while the self-motions over Point 2 did not. Both self-motions are diffeomorphic to a circle, but they are not homotopic. ${ }^{2}$ That is, the self-motions of Point 1 can not 
be continuously deformed on the surface of the configuration space torus to the self-motion of Point 2 . Self-motions which are homotopic to each other form a homotopy class. In other words, all self-motions which are "wrapped around" the configuration space torus in a similar way are members of the same homotopy class. The notion of a homotopy class has previously been used in [Baillieul] to characterize different redundancy resolution paths.

When $r=1$, the homotopy classes of the self-motion manifolds can be interpreted as elements in the fundamental group of $\mathcal{C}$. The fundamental group of an $n$-torus is $\mathbf{Z} \times \mathbf{Z} \times$ $\cdots \times \mathbf{Z}[11]$. Therefore, the homotopy class of a self-motion can be specified by an $n$-tuple of integers $\left(I_{1}, I_{2}, \cdots, I_{n}\right)$, where $I_{j}$ represents how many integral times the self-motion manifold wraps around the $j^{\text {th }}$ configuration space generator. When the relative degree of redundancy is greater than 1 , there does not appear to be a simple way to characterize the homotopy classes of self-motions.

\subsection{Coregular Self-Motions, $\mathcal{C}$-Bundles, and Homo- topy Maps}

For redundant manipulators, when $\mathrm{x}$ is a critical value, the kinematic preimage may contain both critical and regular points (thereby enabling singularity ayoidance). Such $\mathbf{x}$ are termed coregular, and the self-motions in the preimage of such points are termed coregular self-motions. A coregular preimage does not properly form a manifold. The structure of coregular self-motions can be investigated by considering the $3 R$ planar manipulator self-motions as the end-effector nears a singularity (which is not a workspace boundary singularity). At the singularity, two self-motions (which are diffeomorphic to circles) join at a point, to form a "bouquet of circles," or a curve which is diffeomorphic to a figure 8 [6]. Only the waist of the figure 8 is a critical point, whereas the other points in the figure 8 are coregular. Analogous, and often more complicated, coregular motions occur for regional and spatial manipulators.

The preimage of an entire critical value manifold (which is formed by computing $f^{-1}(\mathbf{x})$ as $\mathbf{x}$ is swept along the critical value manifold) is a continuous surface, termed a coregular surjace. Coregular surfaces are not manifolds, but by virtue of their dimension, they can separate the configuration into disjoint regions, and in this regard they are analogous to the critical point surfaces of non-redundant manipulators. Figure 10 illustrates all of the coregular surfaces for the $3 R$ manipulator of Figure 4. In [6] it is shown that the coregular surfaces divide the configuration space into disjoint regions which are fiber bundles. In this context, a fiber bundle is a collection of homotopic self-motion manifolds. Further, these fiber bundles are preimages of workspace subregions bounded by critical values.

Definition: A region in $\mathcal{C}$ bounded by a coregular

2 Two maps, $f_{0}: X \rightarrow Y$ and $f_{1}: X \rightarrow Y$ are homotopic if there exists a smooth map, $F: X \times I \rightarrow Y$ such that $F(x, 0)=f_{0}(x)$ and $F(x, 1)=f_{1}(x)$. In other words, $f_{0}$ can be deformed to $f_{1}$ through a smoothly evolving family of maps. surface is termed a $\mathcal{C}$-bundle. The forward kinematic mapping of a $\mathcal{C}$-bundle is termed a $\mathcal{W}$-sheet. A region in $W$ bounded by critical value manifolds is termed a $\mathcal{W}$-cover, and consists of the union of one or more $\mathcal{W}$-sheets [6]. The preimage of a $\mathcal{W}$-cover is the union of one or more $\mathcal{C}$-bundles.

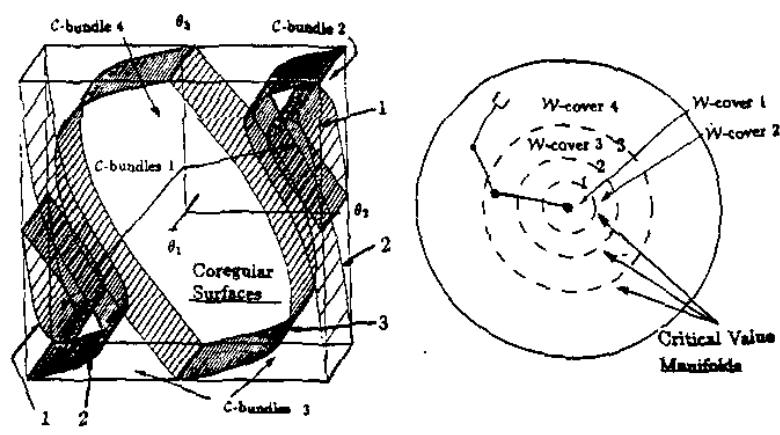

Figure 10: 3R Manipulator Coregular Surfaces

The number of $\mathcal{C}$-bundles over a $\mathcal{W}$-cover is equivalent to the number of distinct self motions in that region of $\mathcal{W}$. In Figure $10, \mathcal{W}$-covers 1 and 3 have two $\mathcal{C}$-bundles in their preimages ( $\mathcal{C}$-bundles 1 and 3 ), indicating the presence of two self-motions in these regions, while $\mathcal{W}$-covers 2 and 4 have only one $\mathcal{C}$-bundle preimage, and one associated selfmotion. The number of distinct self-motions is constant in a $\mathcal{W}$-cover and only varies when crossing a critical value surface into another $W$-cover. Further, the homotopy class of all self-motions in a $\mathcal{C}$-bundle is the same. Thus, the interior critical value surfaces can be used to define a self-motion homotopy map which encapsulates the variations in homotopy class and number of self-motions across the workspace. As another example, the singularities of the $4 R$ regional manipulator divide the workspace interior into two disjoint homotopy regions. Figure 11 shows a cross section of the $4 R$ manipulator workspace, in a plane containing joint axis 1 . In Region \#1, there are 4 self-motions in which joint 3 undergoes a net $2 \pi$ rotation, while in Region \#2 there are also 4 self-motions in which joint 1 undergoes a net $2 \pi$ rotation in this region. Homotopy maps are useful for global planning operations since the number of possible self-motions and their homotopy class are useful information for planning resource allocation and redundancy resolution feasibility. More importantly, interesting physical phenomena occur at the homotopy class boundaries.

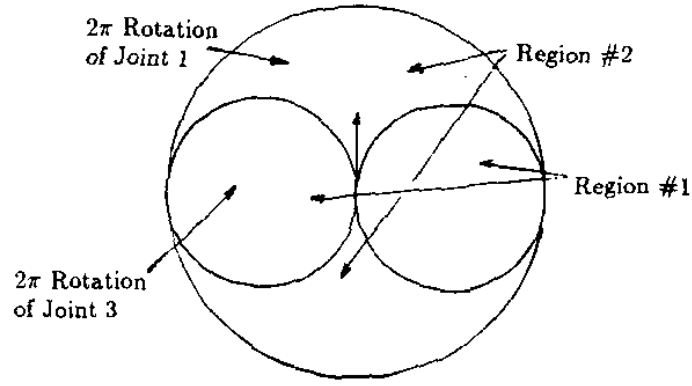

Figure 11: $4 R$ Manipulator Self-Motion Homotopy Map 


\subsection{Self-Motion Homotopy Variations and Algorith-} mic Singularities

All self-motions in a $\mathcal{C}$-bundle are homotopic, implying that the physical motion of the links in a self-motion are similar when the end-effector remains within one $\mathcal{W}$-sheet. However, when crossing co-regular surfaces in $\mathcal{C}$, the self-motion homotopy class can change, leading to a change in the gross physical motion of the links. For example, consider a $7 R$ manipulator which is formed by adding a $3 R$ wrist to the $4 R$ regional manipulator (additional kinematic parameters $\alpha_{4}=\pi / 2, \alpha_{5}=-\pi / 2, \alpha_{6}=\pi / 2, a_{5}=a_{6}=a_{7}=0$, $d_{5}=d_{6}=0, d_{7}$ finite). In most parts of the workspace, the self-motion consists of a rotation of the plane containing links 3 and 4 about the line between the shoulder and the wrist. However, when axis 7 aligns with link 4, axes 1, 2, 3 , and 4 are locked, and the self-motion manifests itself as a self-motion of wrist joint 5 , which is physically very different from the nominal self-motion. This behavior occurs because this configuration is part of a coregular self-motion. At the singularity associated with this coregular self-motion, two radically different self-motions, corresponding to a wrist self-motion and a regional structure self-motion, are joined. This physical effect has two important ramifications. First, the homotopy variation can lead to reduction in a manipulator's ability to satisfy redundancy resolution objectives. In this case, the capability to avoid obstacles is suspended in this configuration, even though the manipulator is not in a singular configuration.

Second, the homotopy variations can lead to algorithmic singularities in some methods of redundant manipulator trajectory planning. In the augmented Jacobian method [14] (similar results hold for the extended Jacobian method of [1]), one defines $r$ additional kinematic objective functions, $\mathrm{G}(\bar{\theta})$, which are to be satisfied using the $r$ redundant degrees of freedom. Instantaneously, the end-effector augmented task vector and the joint variables are related through and augmented Jacobian, $\mathbf{J}_{a}$ :

$$
\left\lfloor\begin{array}{c}
\dot{\mathbf{x}} \\
\dot{\mathbf{G}}(\bar{\theta})
\end{array}\right\rfloor=\left[\begin{array}{c}
\mathbf{J}(\bar{\theta}) \\
\partial G(\bar{\theta}) / \partial \bar{\theta}
\end{array}\right] \dot{\bar{\theta}}=\left[\begin{array}{c}
\mathbf{J}(\bar{\theta}) \\
\mathbf{J}_{\mathbf{G}}(\bar{\theta})
\end{array}\right]=\mathbf{J}_{a}(\bar{\theta})
$$

The augmented Jacobian can be inverted and the inverse of (5) integrated to compute joint trajectories which track an end-effector trajectory while satisfying $\mathbf{G}(\bar{\theta})$. However, this method fails when $\mathbf{J}_{a}$ becomes singular. $\mathbf{J}_{a}$ can lose rank when 1) $\mathbf{J}(\mathbf{q})$ loses rank-this is a kinematic singularity; 2) when $\mathbf{J}_{\mathbf{G}}$ loses rank; 3 ) when the null space of $\mathbf{J}$ and the null space of $\mathbf{J}_{\mathbf{G}}$ intersect: $N(\mathbf{J}) \cap N\left(\mathbf{J}_{\mathbf{G}}\right) \neq \emptyset$. In the third case, both submatrices of the extended Jacobian have full rank, but are not independent. The coregular self-motion example given above can lead to an algorithmic singularity if the redundancy resolution objective is obstacle avoidance. In the coregular configuration, $\mathbf{J}$ is full rank, and its null space is the wrist self-motion. Similarly, the null space of $\mathbf{J}_{\mathrm{G}}$ is also the wrist self-motion, since $\mathrm{it}$ does not change the geometric profile of the manipulator, which is necessary for obstacle avoidance. Consequently, condition (3) is satisfied, and this configuration leads to an algorithmic singularity.

\section{Conclusion}

This paper briefly reviewed a global framework for kinematic analysis which could be useful for higher level manipulator motion planning and control. Local trajectory generation algorithms are often useful because of efficiency. However, certain subtle kinematic and geometric manipulator properties can pose difficulties for local methods, and information on global manjpulator capabilities is sometimes needed for intelligent planning of highly dextrous tasks, and for assisting local methods when they fail.

\section{REFERENCES}

[1] J. Baillieul, "Kinematic Programming Alternatives for Redundant Manipulators," Proceedings of the IEEE Intcrnational Conference on Robotics and Automation, St. Louis, MO, March 25-28, 1985, pp. $722-$ 728 .

[2] J. Baillieul and D. P. Martin, "Issues in the Control of Kinematically Redundant Mechanisms," proceedings of the Nato Workshop on Redundancy in Robotics: Sensing, Design, and Control, Salo, Italy, June 26-July 1, 1988, Springer Verlag.

[3] D. R. Baker and C.W. Wampler, "On the Inverse Kinematics of Redundant Manipulators," The International Journal of Robotics Re. search, vol. 7, no.2, March/A pril, 1988, pp. 3-21.

[4] P. Borrel and A. Liegeois, "A Study of Manipultor Inverse Kinematic Solutions with Applications to Trajectory Planning and Workspace Determination, Proceedings of the IEEE International Conference on Robotics and Automation, San Francisco, Ca, April 1986, pp. $1180-$ 1185 .

[5] J.W. Burdick, "A Classification of 3R Manipulator Singularities and Geometries," Robotics and Mechanical Systems Report no. RNIS89-02, Dept. of Engineering and Applied Science, California institute of Technology, October 1989.

[6] J. W. Burdick, "Kinematic Analysis and Design of Redundant Rolot Manipulators," Stanford Computer Science Report no. STAN-CS-881207.

[7] J.W. Burdick, "On the Inverse Kinematics of Redundant Manipulators: Characterization of the Self-Motion Manifolds. "Prociedings of 1989 IEEE International Conference on Robotics and Automation. May 15-19, 1989, Scottsdale, AZ.

[8] D. H. Gottlieb, "Robots and Topology," Proceedings of the IEEE International Conference on Robotics and Automation, A pril $\bar{i}-10$. $198 \bar{i}$. San Francisco, CA, vol. 3, pp. 1689-1691.

[9] V. Guillemiı and A. Pollack, Differential Topology, Prentice-Hall. Inc., Englewood Cliffs, New Jersey, 1974 .

[10] C.A. Klein and C.H. IIuang, "Review of the Pseudoinverse for Control of Kinematically Redundant Manipulators." IEEE Trans. on Systems, Man and Cybernetics, March, 1983.

[11] W.S. Massey, Algebraic Topology: An Introduction Springer-Verlag. New York. 1967.

[12] D.K. Pai, "Singularity, Uncertainty, and Compliance of Robot Manipulators," Ph.D. Thesis, Dept. of Mechanical Engineering, Cornell University, Ithaca, N.Y., May 1988.

[13] T. Shamir and Y. Yomdin, "Repeatibility of Redundant Manipulators: Mathematical Solution of the Problem." IEEE Trans. on Automatic Control, vol. 33, no. 11, Nov., 1988, pp. 1004-1009.

[14] H. Seraji, "Configuration Control of Redundant Manipulators." Proceedings of the NATO Advanced Research W'orkshop on Robots with Redundancy: Design, Sensing, and Control, Salo. Italy. June 26-July 1, 1988, Springer-V'eriag.

[15] C. Wampler, "On the Inverse Kinematics of Redundant Manipulators," Proceedings of the Nato Workshop on Redundancy in Robotics. Sensing, Design, and Control, Salo. Italy. June 20. July 1. 1988. Springer| V'erlag. 